\title{
Analysis of chaotic saddles in high-dimensional dynamical systems: The Kuramoto-Sivashinsky equation
}

\author{
Erico L. Rempel and Abraham C.-L. Chian \\ National Institute for Space Research (INPE) and World Institute for Space Environment Research \\ (WISER), P. O. Box 515, 12227-010 São José dos Campos-SP, Brazil \\ Elbert E. N. Macau and Reinaldo R. Rosa \\ National Institute for Space Research (INPE), P.O. Box 515, 12227-010 São José dos Campos-SP, Brazil
}

(Received 3 March 2004; accepted 19 April 2004; published online 22 June 2004)

\begin{abstract}
This paper presents a methodology to study the role played by nonattracting chaotic sets called chaotic saddles in chaotic transitions of high-dimensional dynamical systems. Our methodology is applied to the Kuramoto-Sivashinsky equation, a reaction-diffusion partial differential equation. The paper describes a novel technique that uses the stable manifold of a chaotic saddle to characterize the homoclinic tangency responsible for an interior crisis, a chaotic transition that results in the enlargement of a chaotic attractor. The numerical techniques explained here are important to improve the understanding of the connection between low-dimensional chaotic systems and spatiotemporal systems which exhibit temporal chaos and spatial coherence. (C) 2004 American Institute of Physics. [DOI: 10.1063/1.1759297]
\end{abstract}

In the past decades chaos theory has been revealed as a powerful way to explain the physics of low-dimensional dynamical systems, that is, dynamical systems with a small number of state variables. However, most problems of practical interest in physics and engineering are described by partial differential equations (PDEs), and it is still unclear in which situations the physical mechanisms responsible for the onset of chaos in low-dimensional systems are applicable to systems described by PDEs. Several authors have tried to develop a dynamical systems theory for high-dimensional dynamical systems, frequently described by sets of coupled ordinary differential equations obtained as approximations to the original PDEs. ${ }^{1-7}$ The simplest approach consists in first "borrowing" the tools developed for low-dimensional dynamical systems and applying them to spatially extended systems in regimes that display characteristics of lowdimensional chaos. Then, it is possible to develop the tools to be used in more complex regimes. In this paper we try to improve the understanding of the connection between low-dimensional chaotic systems and a certain class of spatiotemporal systems: the one that exhibits temporal chaos and spatial coherence. We focus on the development of a methodology to study the role played by nonattracting chaotic sets called chaotic saddles in chaotic transitions of high-dimensional dynamical systems.

\section{INTRODUCTION}

Chaotic saddles are responsible for important nonlinear phenomena, such as chaotic transients, ${ }^{8-11}$ chaotic scattering, ${ }^{12,13}$ and fractal basin boundaries. ${ }^{14-16}$ It has also been shown that chaotic saddles have crucial importance in chaotic transitions known as interior crises in low-dimensional ${ }^{17-20}$ and high-dimensional ${ }^{21}$ dynamical sys- tems. An interior crisis is a sudden and dramatic increase in the size of a chaotic attractor as a control parameter of the system passes through a critical value., ${ }^{8,9,22,23}$ We describe a novel technique that uses the numerically computed stable manifold of a chaotic saddle to characterize the homoclinic tangency responsible for an interior crisis in a PDE.

For this study we have chosen the KuramotoSivashinsky (KS) equation as our model equation. The KS equation is one of the most widely studied nonlinear partial differential equations to model reaction-diffusion systems. It was named after its derivation as a phase equation for the complex Ginzburg-Landau equation, presented by Kuramoto and Tsuzuki, ${ }^{24}$ and as a model for hydrodynamical instability in laminar flame fronts, presented by Sivashinsky. ${ }^{25}$ However, the KS equation had been previously obtained as a model for the nonlinear saturation of drift waves associated with the oscillation of plasma particles trapped in magnetic wells created by the inhomogeneous magnetic field of a tokamak. ${ }^{26}$ Since then, the KS equation has been used to study Rayleigh-Bénard convection and flow of a viscous fluid down a vertical plane, ${ }^{27}$ nonlinear saturation of Rayleigh-Taylor instability in thin films, ${ }^{28}$ and the dynamics of bright spots formed by self-focusing of a laser beam. ${ }^{29} \mathrm{We}$ choose a regime in which the dynamics of the KS equation is chaotic in time, but remains coherent in space. This enables us to develop numerical schemes for analyzing highdimensional dynamical systems. It is known that for certain choices of the control parameters, the KS dynamics can become chaotic in both space and time. In such regimes, very different bifurcations and nonlinear mechanisms may appear. We believe the numerical investigation conducted in this paper can be a guide to extend the study of the KS equation to the spatiotemporal chaos regime.

This paper develops as follows. In Sec. II the KS equation is numerically solved using the Galerkin method. In Sec. III two algorithms for finding chaotic saddles are described. 
In Sec. IV we study the presence of chaotic saddles in the KS equation. In Sec. V we present our method to characterize a high-dimensional homoclinic tangency using the stable manifold of a chaotic saddle. Section VI describes specific details regarding the numerical procedures for finding chaotic saddles in the KS equation. The conclusions and final commentaries are given in Sec. VII.

\section{THE KURAMOTO-SIVASHINSKY EQUATION} as $5,21,23,26$

$$
\partial_{t} u=-\partial_{x}^{2} u-\nu \partial_{x}^{4} u-\partial_{x} u^{2},
$$

where $\nu$ is a "viscosity" damping parameter and we assume that $u(x, t)$ is subject to periodic boundary conditions

$$
u(x, t)=u(x+2 \pi, t) .
$$

To obtain the numerical solution of Eq. (1) we use the Galerkin method, ${ }^{30}$ by applying a Fourier decomposition for the function $u(x, t)$

$$
u(x, t)=\sum_{k=-\infty}^{\infty} b_{k}(t) e^{i k x} .
$$

A substitution of Eq. (3) into Eq. (1) yields

$$
\begin{aligned}
\frac{\partial}{\partial t}\left(\sum_{k=-\infty}^{\infty} b_{k}(t) e^{i k x}\right) \\
=-\frac{\partial^{2}}{\partial x^{2}}\left(\sum_{k=-\infty}^{\infty} b_{k}(t) e^{i k x}\right)-\nu \frac{\partial^{4}}{\partial x^{4}}\left(\sum_{k=-\infty}^{\infty} b_{k}(t) e^{i k x}\right) \\
-\frac{\partial}{\partial x}\left(\sum_{k=-\infty}^{\infty} \sum_{m=-\infty}^{\infty} b_{k}(t) b_{m}(t) e^{i x(k+m)}\right) .
\end{aligned}
$$

After solving the derivatives in Eq. (4), one obtains

$$
\begin{aligned}
\sum_{k=-\infty}^{\infty} \dot{b}_{k} e^{i k x}= & \sum_{k=-\infty}^{\infty} k^{2} b_{k} e^{i k x}-\nu \sum_{k=-\infty}^{\infty} k^{4} b_{k} e^{i k x} \\
& -\sum_{k=-\infty}^{\infty} \sum_{m=-\infty}^{\infty} i(k+m) b_{k} b_{m} e^{i x(k+m)}
\end{aligned}
$$

Next, multiply Eq. (5) by $e^{-i l x}$, with $l=-\infty, \ldots, \infty$, and integrate in the spatial domain

$$
\begin{aligned}
\int_{0}^{2 \pi} \sum_{k=-\infty}^{\infty} \dot{b}_{k} e^{i x(k-l)} d x \\
=\int_{0}^{2 \pi} \sum_{k=-\infty}^{\infty} k^{2} b_{k} e^{i x(k-l)} d x \\
\quad-\int_{0}^{2 \pi} \nu \sum_{k=-\infty}^{\infty} k^{4} b_{k} e^{i x(k-l)} d x \\
-\int_{0}^{2 \pi} \sum_{k=-\infty}^{\infty} \sum_{m=-\infty}^{\infty} i(k+m) \\
\quad \times b_{k} b_{m} e^{i x(k+m-l)} d x .
\end{aligned}
$$

Each integral of the exponential functions in Eq. (6) vanishes except when the exponent is zero. The three first exponents are zero when $k=l$. For the nonlinear term the exponent is null when $k=l-m$. After solving the integrals, Eq. (6) becomes

$$
\dot{b}_{k}=\left(k^{2}-\nu k^{4}\right) b_{k}-i k \sum_{m=-N}^{N} b_{m} b_{k-m}, \quad k=-N, \ldots, N,
$$

where $N$ is the truncation order.

The coefficients $b_{k}$ in Eq. (7) are complex. We can simplify our analysis by restricting to the subspace of odd solutions, $u(x, t)=-u(-x, t)$. It is possible to prove that if an initial condition $u(x, 0)$ is an odd function, the solution of Eq. (1) is odd for all time ${ }^{2}$

$$
u(x, t)=-u(-x, t), \quad \forall x, t .
$$

The Fourier transform of an odd function has purely imaginary coefficients, so we can represent odd functions by assuming that $b_{k}(t)$ are purely imaginary, setting

$$
b_{k}=-\frac{1}{2} i a_{k},
$$

where $a_{k}$ are real numbers. After substituting Eq. (9) into Eq. (7), we obtain

$$
\begin{gathered}
\dot{a}_{k}=\left(k^{2}-\nu k^{4}\right) a_{k}-\frac{k}{2} \sum_{m=-N}^{N} a_{m} a_{k-m}, \\
k=-N, \ldots, N .
\end{gathered}
$$

Equation (10) contains unnecessary operations. Since $u(x, t)$ is real, $-i a_{k}=i a_{-k}$, and it is not necessary to compute the modes with negative $k$. Besides that, $a_{k}=0$ for $|k|$ $>N$, and some operations in the nonlinear term can be dropped. Thus Eq. (10) can be written in the form

$$
\begin{aligned}
\dot{a}_{k}= & \left(k^{2}-\nu k^{4}\right) a_{k}+\frac{k}{2}\left(\sum_{m=k-N}^{-1} a_{-m} a_{k-m}-\sum_{m=1}^{k-1} a_{m} a_{k-m}\right. \\
& \left.+\sum_{m=k+1}^{N} a_{m} a_{m-k}\right),
\end{aligned}
$$

with $k=1, \ldots, N$.

\section{ALGORITHMS}

In this section two algorithms for detecting chaotic saddles are briefly reviewed: the sprinkler and the proper interior maximum (PIM) triple methods. The algorithms are described for discrete-time maps. Thus, for differential equations the trajectories mentioned in the following sections refer to the iterations of a Poincaré map. ${ }^{31}$

\section{A. The sprinkler method to find chaotic saddles and their invariant manifolds}

Associated to a chaotic saddle, there are stable and unstable manifolds. The stable manifold is the set of points that converge to the chaotic saddle in forward time dynamics; the unstable manifold is the set of points that converge to the chaotic saddle in the time reversed dynamics. The chaotic saddle lies on the intersection of its stable and unstable manifolds, ${ }^{32,33}$ and contains an infinite number of unstable 
periodic orbits (UPOs). In this section we describe a simple routine to obtain an approximation to a chaotic saddle and its stable and unstable manifolds.

Consider a system with an attractor $A$ and a region $R$ in the phase space containing a chaotic saddle $\Lambda$ and no attractor (a so-called restraining region). The trajectories of all initial conditions in $R$ will eventually leave $R$ and converge to attractor $A$, except for initial conditions lying on the stable manifold of $\Lambda$, which is a set of measure zero. Initial conditions close to the stable manifold are first attracted to $\Lambda$ and stay close to its neighborhood for some time, before they are repelled, following its unstable manifold. The closer an initial condition is to the stable manifold of $\Lambda$, the longer its transient time before leaving $R$. These facts lead to the sprinkler algorithm ${ }^{34,35}$ to find chaotic saddles and their manifolds. Define a grid with $N_{0}$ points uniformly distributed in $R$. Obtain the exit time of each point, that is, the time it takes to leave $R$. Those initial conditions with an exit time larger than some specified time $t_{c}$ constitute an approximation to the stable manifold of $\Lambda$, and their last iterations before leaving $R$ approximate the unstable manifold. The points at some time $\bar{t}=\xi t_{c}$ are the approximation to the chaotic saddle. The values of $t_{c}$ and $\bar{t}$ must be chosen after some trial and error, but usually $t_{c}$ must be large compared to the average exit time $\tau$ and $\xi=1 / 2$ seems to work fine in most systems, according to Hsu et al. ${ }^{35}$ Let $N_{t}$ be the number of trajectories that are still in the restraining region after $t$ iterates. $N_{t}$ typically decreases exponentially with time $t$ at a rate $1 / \tau^{10,35}$

$$
N_{t}=N_{0} \exp (-t / \tau)
$$

\section{B. The PIM triple method to find chaotic saddles}

In the sprinkler algorithm, a chaotic saddle $\Lambda$ is approximated by a series of fragments of different trajectories. The PIM triple method, first presented by Nusse and Yorke, ${ }^{32}$ finds a single trajectory which stays close to the chaotic saddle for an arbitrarily long time.

The PIM triple routine receives as an input a pair of points $a$ and $b$ such that the line segment $[a, b]$ intersects or "straddles" the stable manifold of $\Lambda$. In this situation, there must, necessarily, be a point $c$ between $a$ and $b$ which is closer to the stable manifold of $\Lambda$ than $a$ and $b$. The exit time $\tau$ is infinite for a point on the chaotic saddle or on its stable manifold, and the exit time tends to infinity as closer is the point to the stable manifold of $\Lambda$. Thus, the exit time of $c, \tau(c)$, is strictly larger than $\tau(a)$ and $\tau(b)$. The triple $(a, c, b)$ is called a proper interior maximum triple, or a PIM triple.

An initial PIM triple can be found by selecting a large interval $[a, b]$ in the restraining region $R$. Given points $a$ and $b$, the algorithm tries to find the point $c$ of larger exit time than $a$ and $b$ inside $[a, b]$. The PIM triple routine divides the interval $[a, b]$ into $N$ subintervals with the same length, delimited by a grid of points along $[a, b]$. After that, the algorithm obtains the exit time of all the grid points and selects three, not necessarily consecutive, points forming a PIM triple $\left(a_{1}, c_{1}, b_{1}\right)$, by checking if the middle point $c_{1}$ has exit time larger than $a_{1}$ and $b_{1}$. Usually, for $N$ not too small there is one or more PIM triples in $[a, b]$. Next, an $\varepsilon$ refinement of the triple $\left(a_{1}, c_{1}, b_{1}\right)$ is created. An $\varepsilon$ refinement of a triple $(a, c, b)$ is a finite set of points $g_{i}$ in $[a, b]$ such that $a=g_{0}<g_{1}<\ldots<g_{N}=b,\left|g_{k}-g_{k+1}\right| \leqslant \epsilon \cdot|a-b|$, and $c=g_{k}$ for some $k, 1 \leqslant k \leqslant N$. Here, $|a-b|$ denotes the distance between $a$ and $b$. The next step is to find a new triple $\left(a_{2}, c_{2}, b_{2}\right)$ in the $\varepsilon$ refinement of $\left(a_{1}, c_{1}, b_{1}\right)$. Then, an $\varepsilon$ refinement of this triple is generated and a new triple $\left(a_{3}, c_{3}, b_{3}\right)$ is selected. This procedure is repeated obtaining successively smaller PIM triples until the Euclidean length of a PIM triple $\left(a_{n}, c_{n}, b_{n}\right)$ is less than some specified value $\delta$, which defines the precision of the approximation. Let $I_{0}$ be the interval $\left[a_{n}, b_{n}\right]$. Then, iterate the interval $I_{0}$, that is, iterate points $a_{n}$ and $b_{n}$ once using the Poincare map, obtaining a new interval $I_{1}$. If the length of $I_{1}$ is less than $\delta$, iterate $I_{1}$, obtaining $I_{2}$. Keep iterating $I_{n}$ until its length is greater than $\delta$. In that case, refine $I_{n}$ using the PIM triple routine, obtaining a new interval $I \leqslant \delta$. The program proceeds iterating $I$ and calling the PIM triple routine every time its length exceeds $\delta$. After each iteration one point from $I$ is plotted, generating a single trajectory called the straddle trajectory, which stays at a distance of the order of $\delta$ from the true chaotic saddle.

The PIM triple method is usually more accurate than the sprinkler method for systems with short transients, as in our case. Thus, in the following sections we use the PIM triple algorithm to find the chaotic saddles, and the sprinkler algorithm is used to estimate their stable manifolds. We always compare the chaotic saddles obtained with both algorithms in order to check our numerical results.

\section{NONLINEAR DYNAMICS ANALYSIS}

The dynamics of the system described by Eq. (11) can be analyzed on a Poincaré section defined by $a_{1}=0$. A trajectory representing the flow of Eq. (11) in a phase space defined by the Fourier modes $a_{k}$, can intersect this Poincaré section in two ways: when $\dot{a}_{1}>0$ (from "left" to "right") or when $\dot{a}_{1}<0$ (from "right" to "left"). We adopt a Poincaré map $P$ defined as the $(N-1)$ dimensional hyperplane given by $a_{1}=0$, with $\dot{a}_{1}>0$, so that a Poincare point is plotted every time the flow of Eq. (11) crosses the hyperplane $a_{1}$ $=0$ from "left" to "right."

We follow Refs. 5 and 23 and solve Eq. (11) with $N$ $=16$, since for our choices of the control parameter $\nu$ the dynamics is qualitatively the same for $N>16$. A typical spatiotemporal pattern of $u(x, t)$, recovered from the values of $b_{k}$ using Eqs. (3) and (9), is plotted in Fig. 1. Note that the dynamics is chaotic in time, but spatially coherent structures are preserved.

For certain values of $\nu$, the trajectories of random initial conditions wander chaotically in a certain region of the phase space for some transient time, before they converge to an attractor. During this chaotic transient, the trajectories are in the vicinity of a chaotic saddle. Figure 2(a) shows the variation of a chaotic saddle (gray) as a function of $\nu$, superimposed by the bifurcation diagram of the attractor (black). For each value of $\nu$, we use the PIM triple algorithm to find 250 Poincaré points of a straddle trajectory at a distance $\delta$ $\approx 10^{-6}$ from the true chaotic saddle, and plot the Fourier 


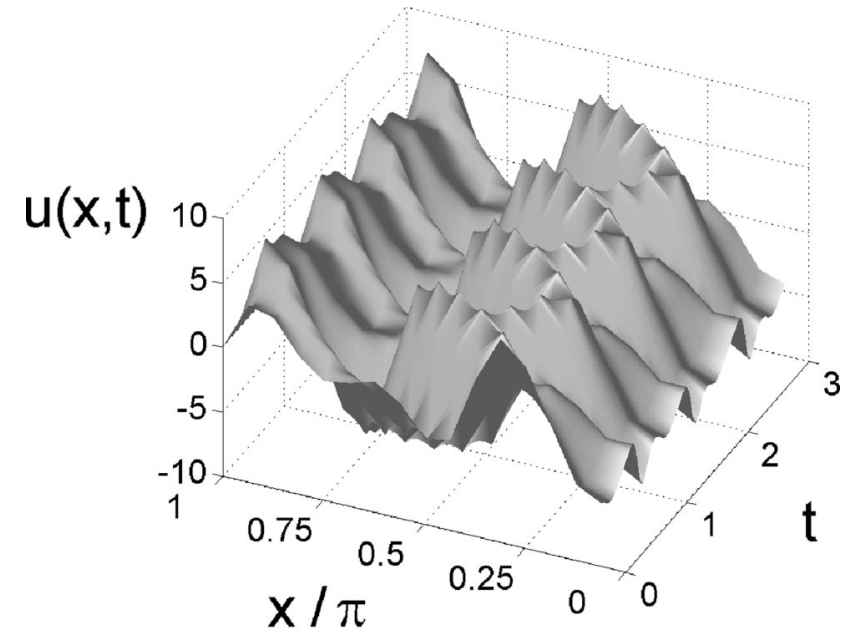

FIG. 1. The spatiotemporal pattern of $u(x, t)$ at $\nu=0.02992006$. The system dynamics is chaotic in time but coherent in space.
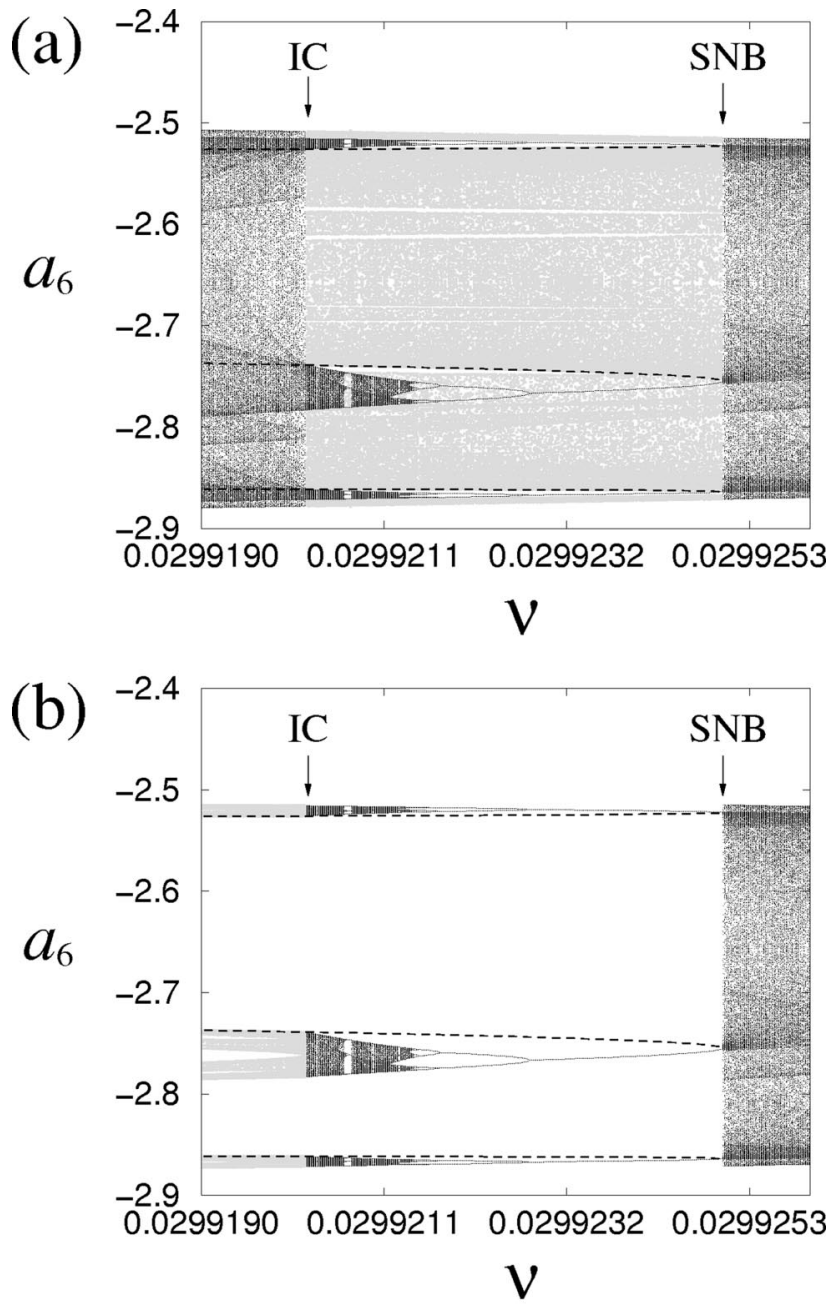

FIG. 2. (a) Variation of $a_{6}$ for the chaotic saddle (gray) as a function of $\nu$, superimposed by the bifurcation diagram of the attractor (black) in a p-3 periodic window. IC denotes interior crisis and SNB denotes saddle-node bifurcation. The dashed lines denote the p-3 mediating unstable periodic orbit. (b) Same as (a), but depicting the conversion of the three-band chaotic attractor into a band chaotic saddle after (to the left of) IC. component $a_{6}$. The bifurcation diagram of the attractor was generated by integrating Eq. (11) and plotting 250 Poincaré points for each value of $\nu$, after dropping the first 100 iterations.

The saddle-node bifurcation (SNB) arrow in Fig. 2(a) points to a saddle-node bifurcation that occurs at $\nu=\nu_{\mathrm{SNB}}$ $\sim 0.02992498$ and marks the beginning of a periodic window. To the right of $\nu_{\mathrm{SNB}}$, initial conditions are attracted to a chaotic attractor. In the saddle-node bifurcation, a pair of period-3 (p-3) stable/unstable periodic orbits is created. As $\nu$ is decreased to the left of $\nu_{\mathrm{SNB}}$, the stable p-3 attractor (solid lines) undergoes a series of period-doubling bifurcations, leading to a chaotic attractor localized in three separate bands in the bifurcation diagram. The dashed lines in Fig. 2(a) denote the Poincare points of the p-3 unstable periodic orbit created at $\nu_{\mathrm{SNB}}$, which, in the Poincare map, consists of three saddle points with their associated stable and unstable manifolds. As reported by Chian et al. ${ }^{23}$ this UPO, hereafter called the mediating unstable periodic orbit (MPO), is responsible for the onset of interior crisis (IC) at $\nu=\nu_{\text {IC }}$ $\sim 0.02992021$ and is found using the Newton method. ${ }^{5,36} \mathrm{At}$ $\nu_{\text {IC }}$, the MPO collides with the three-band chaotic attractor. After the collision the chaotic attractor is suddenly enlarged. We call the region occupied by the attractor throughout the periodic window, the band region $(B)$, and the region occupied by the chaotic saddle, the surrounding region $(S) .{ }^{17-19}$ Since the chaotic saddle shown in Fig. 2(a) lies in the surrounding region, it is called the surrounding chaotic saddle (SCS). It has been shown that for dissipative dynamical systems described by discrete maps, the stable manifold of MPO determines the boundary between the band region and the surrounding region. ${ }^{17-19}$

After colliding with the mediating unstable periodic orbit, the band chaotic attractor loses its stability and is converted into a chaotic saddle localized in the $B$ region, immersed in the abruptly enlarged chaotic attractor. In contrast with the surrounding chaotic saddle, this newly created chaotic saddle is called band chaotic saddle (BCS). In Fig. 2(b) we plot part of the same bifurcation diagram of Fig. 2(a) prior to IC $\left(\nu>\nu_{\mathrm{IC}}\right)$, and after IC $\left(\nu<\nu_{\mathrm{IC}}\right)$ we plot the variation of the newly created BCS.

It is important to stress that, although in Fig. 2(a) we plot the surrounding chaotic saddle only inside the periodic window (between SNB and IC), it is actually present in the whole bifurcation diagram. For $\nu\left\langle\nu_{\mathrm{IC}}\right.$ and $\nu>\nu_{\mathrm{SNB}}$ the chaotic saddle is embedded in the chaotic attractor. As an example, Fig. 3 shows a three-dimensional projection $\left(a_{1}, a_{10}\right.$, $a_{16}$ ) of a chaotic saddle defined in the 15-dimensional Poincaré hyperplane at $\nu=0.029925>\nu_{\mathrm{SNB}}$, to the right of the saddle-node bifurcation in Fig. 2(a). This chaotic saddle is a continuation of the surrounding chaotic saddle shown in Fig. 2(a). The chaotic saddle is not a continuous line. It has many gaps, most of which are not visible in Fig. 3 due to their small size.

For the range of the damping parameter $\nu$ used in Fig. 2 and the spatial system size $L=2 \pi$ the KS equation presents some features of low-dimensional dynamical systems. In the chaotic regime the system exhibits only one positive Lyapunov exponent, ${ }^{31}$ which means that there is only one 


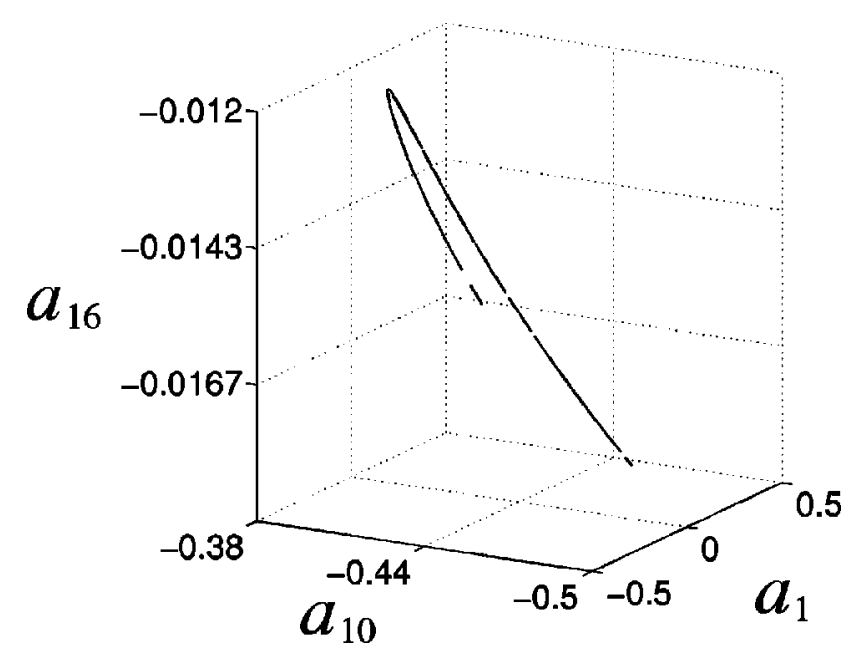

FIG. 3. Three-dimensional projection $\left(a_{1}, a_{10}, a_{16}\right)$ of the chaotic saddle defined in the 15-dimensional Poincare hyperplane just before the saddlenode bifurcation, at $\nu=0.029925$.

expanding direction. An estimation of the fractal dimension of the chaotic attractor at $\nu=0.02992006$ using the KaplanYorke conjecture ${ }^{37}$ results in the dimension $D \approx 2.08$, which implies that in the Poincare section the chaotic attractor is a set of dimension $D_{p}=D-1 \approx 1.08$, and hence the attractor is a low-dimensional set embedded in a high-dimensional phase space. This can be seen in Fig. 4, which shows a three-dimensional projection $\left(a_{1}, a_{10}, a_{16}\right)$ of the Poincaré points of the enlarged chaotic attractor [(ECA) light line] after crisis $(\nu=0.02992006)$, superimposed by the threeband chaotic attractor [(BCA) dark lines] at crisis $(\nu$ $=0.02992021$ ). Note the similarity of the enlarged chaotic attractor with the surrounding chaotic saddle shown in Fig. 3.

The interior crisis occurs when the band chaotic attractor collides with the mediating unstable periodic orbit and with its stable manifold. In Sec. V we show that a homoclinic tangency occurs at this crisis, i.e., the tangency between the stable and unstable manifolds of MPO. After the crisis, it is

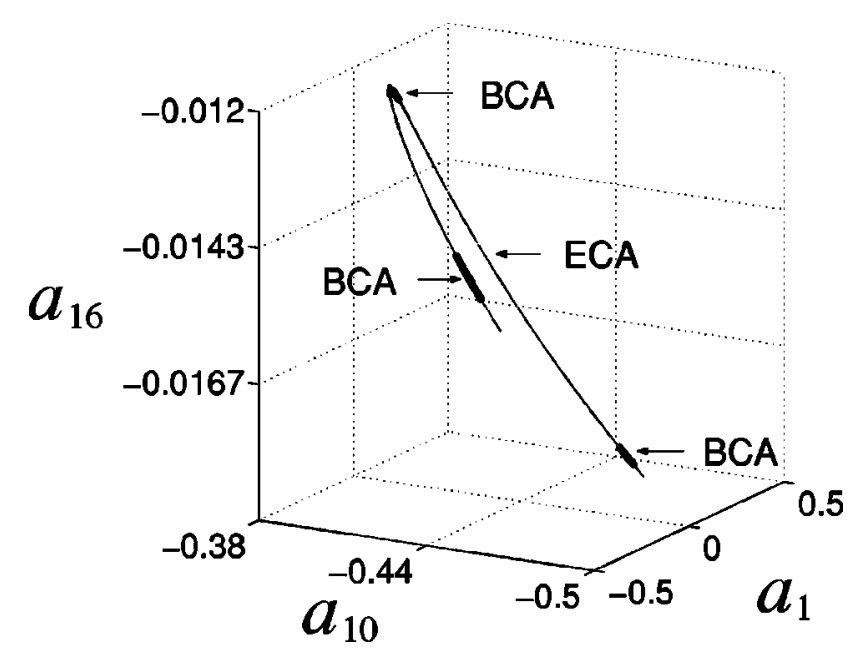

FIG. 4. Three-dimensional projection $\left(a_{1}, a_{10}, a_{16}\right)$ of the ECA (light line) defined in the 15-dimensional Poincare hyperplane after crisis at $\nu$ $=0.02992006$, superimposed by the three-BCA (dark lines) at crisis $(\nu$ $=0.02992021$ ).
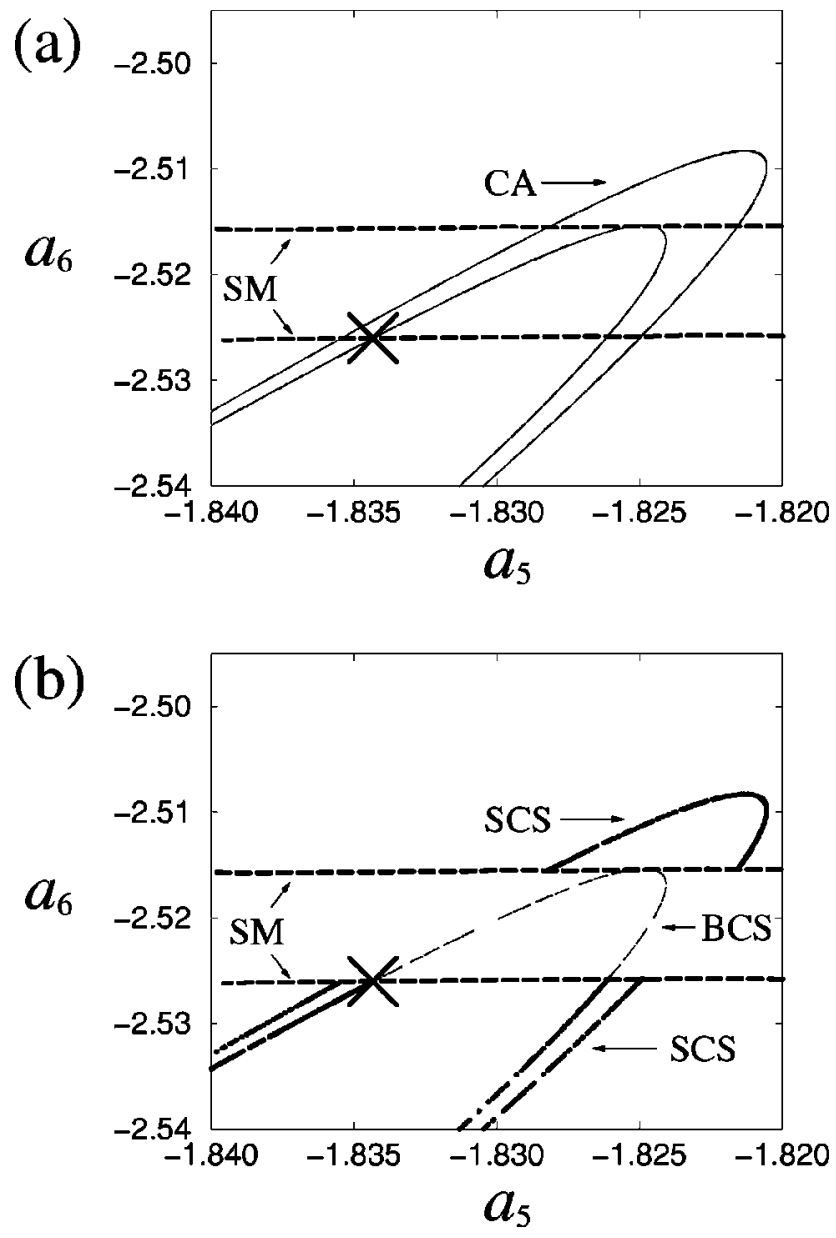

FIG. 5. (a) Upper branch of the CA after the interior crisis, at $\nu$ $=0.02992006$. The dashed lines indicate segments of the SMs of the mediating unstable periodic orbit (cross); (b) the BCS (light lines) and the SCS (dark lines) that compose the chaotic attractor shown in (a).

still possible to determine the $B$ and $S$ regions, which are separated by the stable manifold of MPO. Figure 5 shows a two-dimensional projection $\left(a_{5}, a_{6}\right)$ of the chaotic sets for $\nu=0.02992006$, after the crisis, around the upper branch of the chaotic attractor shown in Fig. 4. Figure 5(a) shows the chaotic attractor (CA) and Fig. 5(b) shows the corresponding $B$ (light lines) and $S$ (dark lines) chaotic saddles. BCS is localized in a region of the phase space previously occupied by the precrisis band chaotic attractor. SCS is the continuation of the precrisis surrounding chaotic saddle. It can be seen from Fig. 5 that the postcrisis $B$ and $S$ chaotic saddles are subsets of the enlarged chaotic attractor. This decomposition of the postcrisis chaotic attractor into two chaotic saddles is important in the study ${ }^{19}$ and control ${ }^{38}$ of crisisinduced intermittency.

\section{VISUALIZING A HIGH-DIMENSIONAL HOMOCLINIC TANGENCY}

In this section we show that at the interior crisis of Fig. 2 the chaotic attractor is tangent to the stable manifold of the surrounding chaotic saddle. We elaborate a method to visualize this tangency in a high-dimensional phase space. Using a suitably chosen grid of initial conditions for the sprinkler 

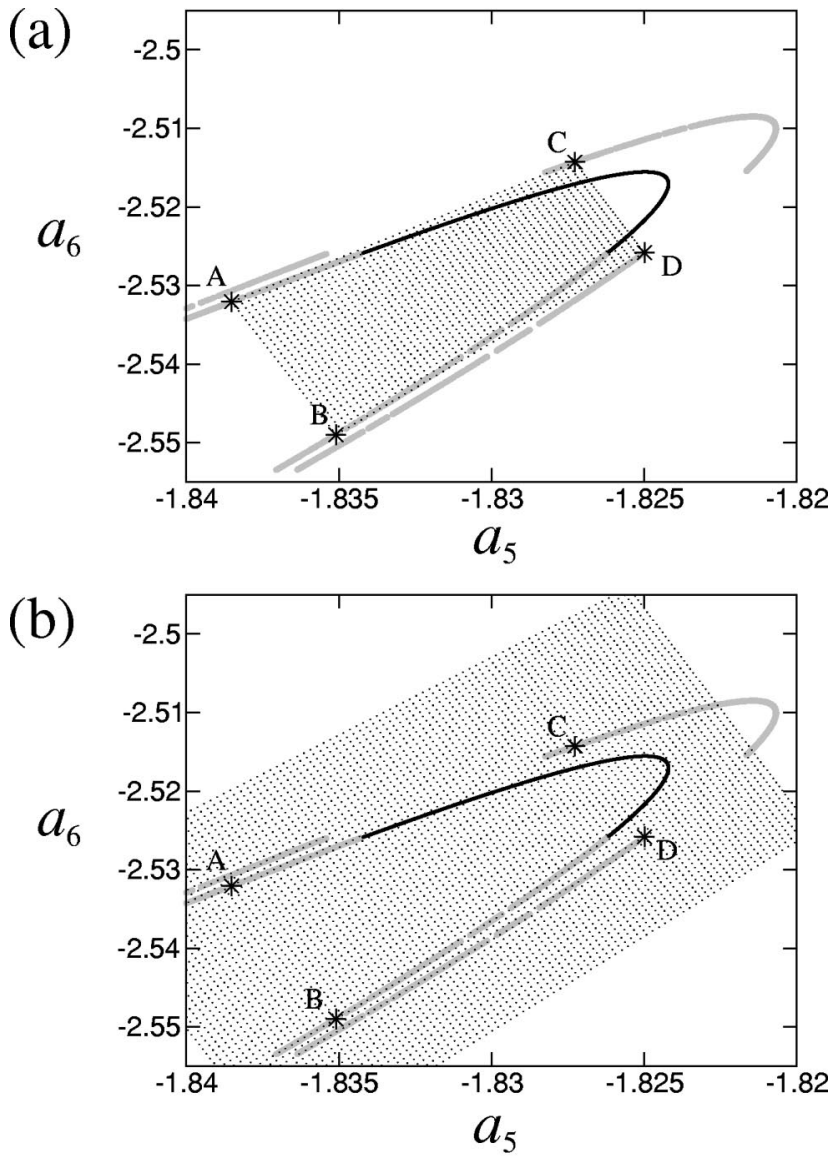

FIG. 6. Grid construction using four points of the chaotic saddle (gray) around the upper branch of the chaotic attractor (black line) at $\nu_{\mathrm{IC}}$ $=0.02992021$. (a) The grid points in the line segment $A B$ are found by linear interpolation. The same is done to find the grid points in segments $C D, A C$, and $B D$. The grid points inside the polygon $A B C D$ are found by linear interpolation of points in the edges. (b) Points outside the polygon $A B C D$ are found by linear extrapolation.

algorithm, we plot a two-dimensional projection of the stable manifold of the surrounding chaotic saddle in the vicinity of the chaotic attractor. We argue that this method can be used to show a homoclinic tangency between the stable and unstable manifolds of MPO. ${ }^{21}$

In a previous work, Lai and Winslow ${ }^{10}$ used the sprinkler method to find the stable manifolds of a chaotic saddle in a high-dimensional phase space of a coupled-map lattice. They used a grid of initial conditions generated by varying the values of two of the state variables while keeping all the other state variables with constant values. This approach is not suited for our application, since we are interested in showing the tangency of the stable manifold of SCS with the chaotic attractor. It is difficult to visualize such a tangency in a high-dimensional phase space, and all the 15 coordinates of each grid point must be carefully chosen. Figure 6 illustrates the process used to create the grid around the upper branch of the band chaotic attractor shown in Fig. 4. First, a set of four points $(A, B, C$ and $D)$ is chosen from the chaotic saddle (gray lines) surrounding the chaotic attractor (black line). These points form a sample from which the grid is interpolated. Figure 6(a) shows a grid of points inside a polygon defined by the vertices $A B C D$. The line segment $[A, B]$ (a)

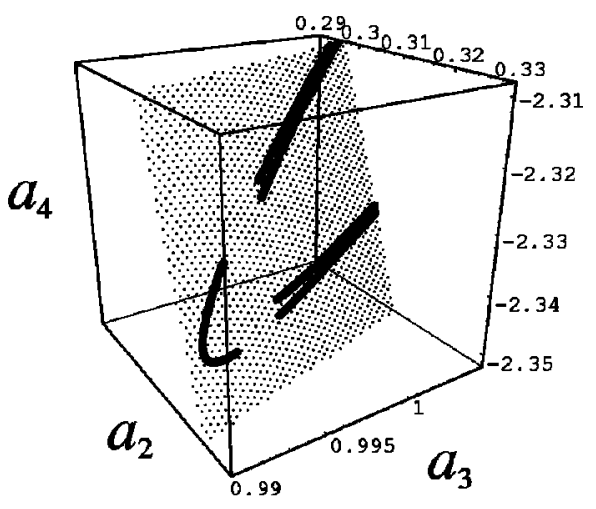

(b)

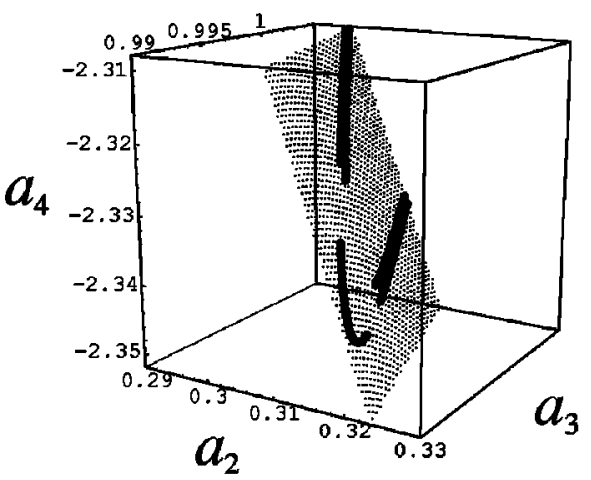

(c)

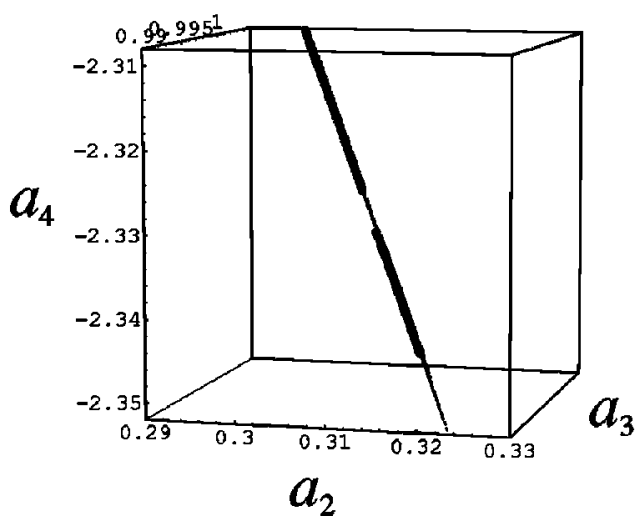

FIG. 7. Three different viewpoints of a three-dimensional projection $\left(a_{2}\right.$, $a_{3}, a_{4}$ ), of the grid along with part of the surrounding chaotic saddle (dark lines) at $\nu_{\mathrm{IC}}=0.02992021$. The grid is close to every point in this part of the chaotic saddle.

is divided into $n$ subintervals delimited by points $p_{i}, i$ $=1, \ldots, n+1$, and the line segment $[C, D]$ is divided in $n$ subintervals delimited by points $q_{i}, i=1, \ldots, n+1$. After that, each line segment defined by a pair of points $\left(p_{i}, q_{i}\right)$ is divided in $m$ subintervals. Linear extrapolation can be used to add points outside the original polygon, as exemplified in Fig. 6(b). For instance, to add ten points above and ten below the segment $[A, B]$, divided in $n$ subintervals, one can use an expression like

$$
\begin{aligned}
& p_{i}=B+i(A-B) / n, \\
& i=-10,-9, \ldots, n+10 .
\end{aligned}
$$

Following this procedure, a grid is built close to one of 
(a)

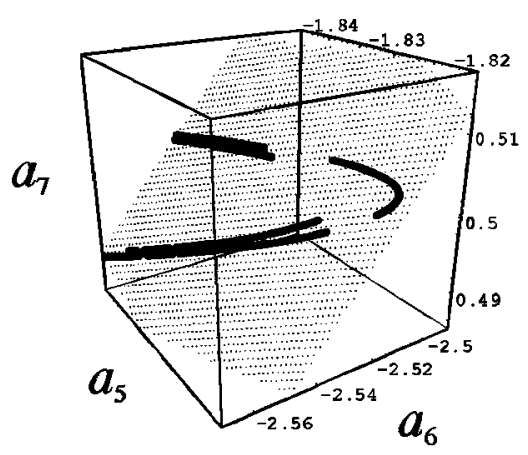

(c)

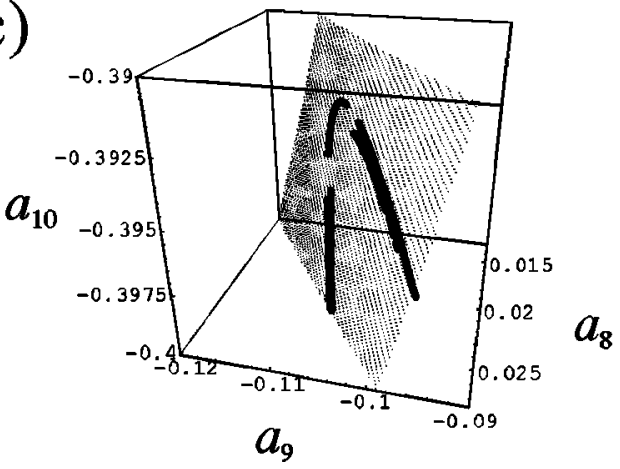

(b)

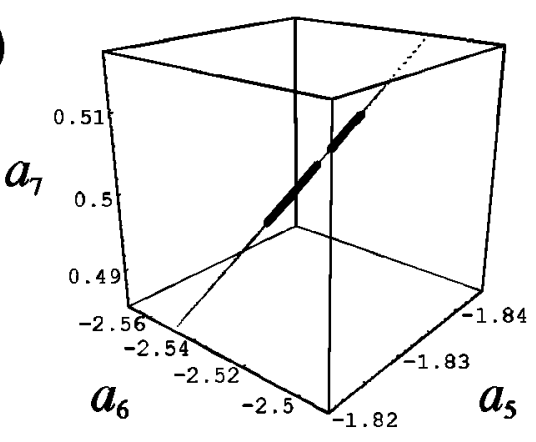

(d)

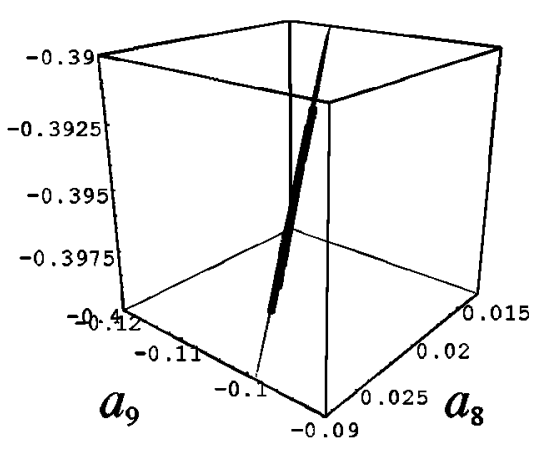

FIG. 8. Two viewpoints of projections $\left(a_{5}, a_{6}, a_{7}\right)$ (a), (b) and $\left(a_{8}, a_{9}, a_{10}\right)$ (c), (d) of the grid with part of the surrounding chaotic saddle (dark lines) at $\nu_{\mathrm{IC}}=0.02992021$.

(a)

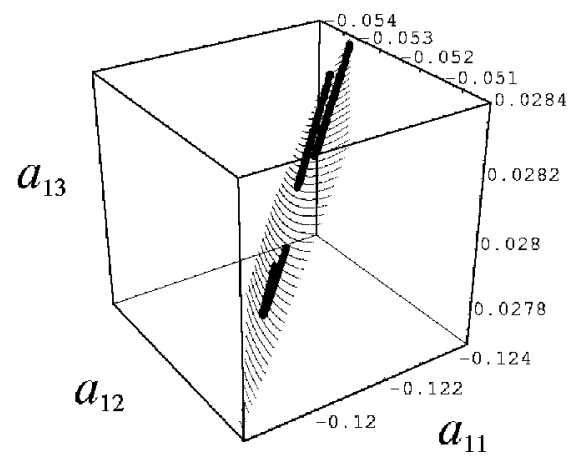

(c)

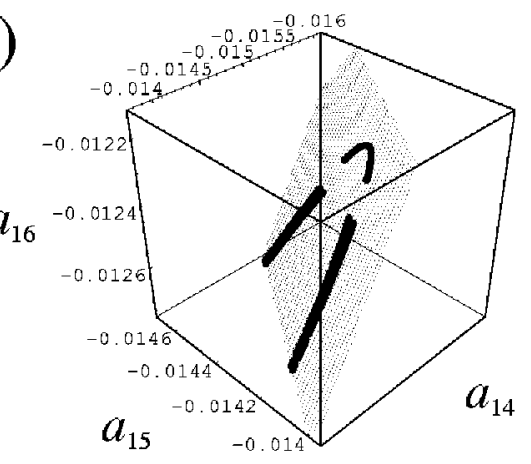

(b)

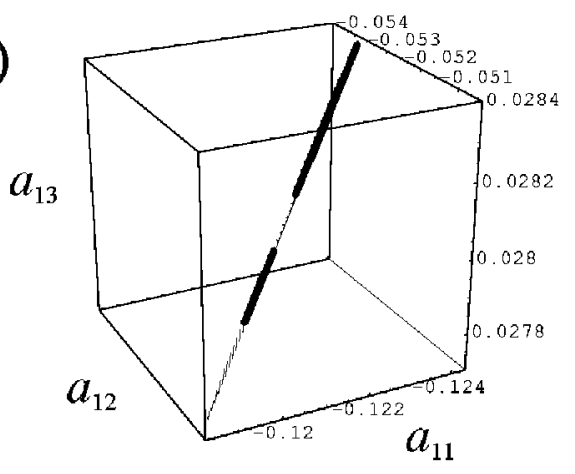

(d)

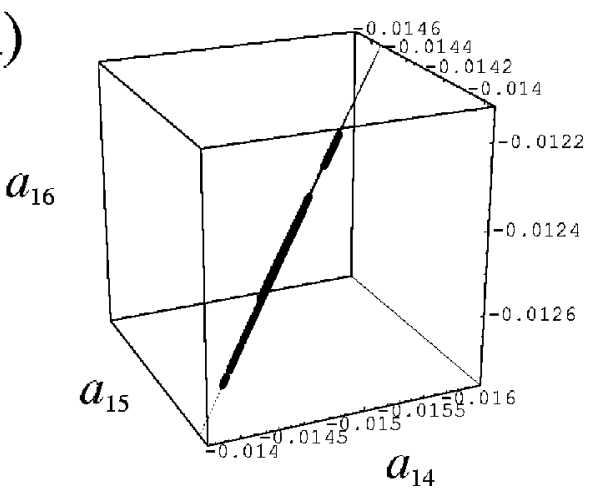

FIG. 9. Two viewpoints of projections $\left(a_{11}, a_{12}, a_{13}\right)$ (a), (b) and $\left(a_{14}, a_{15}, a_{16}\right)$ (c), (d) of the grid with part of the surrounding chaotic saddle (dark lines) at $\nu_{\mathrm{IC}}=0.02992021$. 
the branches of the chaotic attractor. This grid will be used to show a cross section of the stable manifold of SCS in the 15 dimensional (15D) Poincaré phase space.

In Fig. 7 part of SCS around the upper branch of the chaotic attractor at $\nu_{\text {IC }}$ is plotted along with the grid generated. The three different views of this three dimensional (3D) projection $\left(a_{2}, a_{3}, a_{4}\right)$ suggest that the grid is approximately tangent to each point on this part of the chaotic set. In order to verify if the same happens in the other coordinates of the phase space, we plot a series of different 3D projections involving all the Fourier modes. These projections, shown in Figs. 8 and 9, reveal that the linear interpolation method is able to define an appropriate grid to study the dynamics in the vicinity of these chaotic sets.

To show a tangency between the chaotic attractor and stable manifold of SCS, to be calculated on the grid of points with the sprinkler algorithm, the grid points must "touch" all the points on the chaotic attractor. In order to estimate the precision of our method for this problem, we compute the average distance between points on the attractor and the grid. The distance can be computed as follows:

(1) Randomly choose a point $p$ from the upper branch of the chaotic attractor;

(2) search for the grid point $q$ closest (with the smallest Euclidean distance) to $p$;

(3) save the distance $\Delta_{i}=\|p-q\|$;

(4) repeat steps $1-3, N$ times;

(5) obtain the average distance between points on the attractor and the grid as

$D=\frac{1}{N} \sum_{i=1}^{N} \Delta_{i}$.

For the grid in Fig. 11(b), which has 575333 points, we find $D=4.20 \times 10^{-5}$ for $N=500$, which can be seen as an estimate for the precision of the grid. The use of finer grids results in a smaller $D$.

Once the grid is defined, the sprinkler method can be readily applied. In order to set the value of $t_{c}$ we first compute the average exit time $\tau$. Given $N_{0}$ initial conditions we compute $N_{t}$, the number of trajectories that are still in the restraining region after $t$ iterates (the restraining region for this problem is discussed in Sec. VI). Figure 10 shows a graph of $\log N_{t}$ versus $t$ at $\nu_{\text {IC }}$, where $N_{0}=10000$ different initial conditions were used. The graph can be fitted with a straight line of slope $\gamma=-3.59 \times 10^{-2} \pm 1.45 \times 10^{-4}$, which gives an average exit time $\tau=-1 / \gamma \approx 27.9$ [see Eq. (12)]. Based on this value we choose $t_{c}=45$, since it should be large compared to $\tau$. Actually, the numerical results are not very sensitive to $t_{c}$ and different values give similar results, as long as they are not too small.

We proceed by obtaining the exit time for each grid point. Points with exit time larger than $t_{c}$ are considered as part of the stable manifold of SCS. In Fig. 11 we plot the upper branch of the CA (light line) with the surrounding chaotic saddle SCS (dark lines) and its stable manifold (dots in the background) for: (a) $\nu=0.0299211$ (before crisis) and (b) $\nu=0.02992021$ (at crisis). The upper Poincare point of the mediating orbit is represented by the cross. Note that

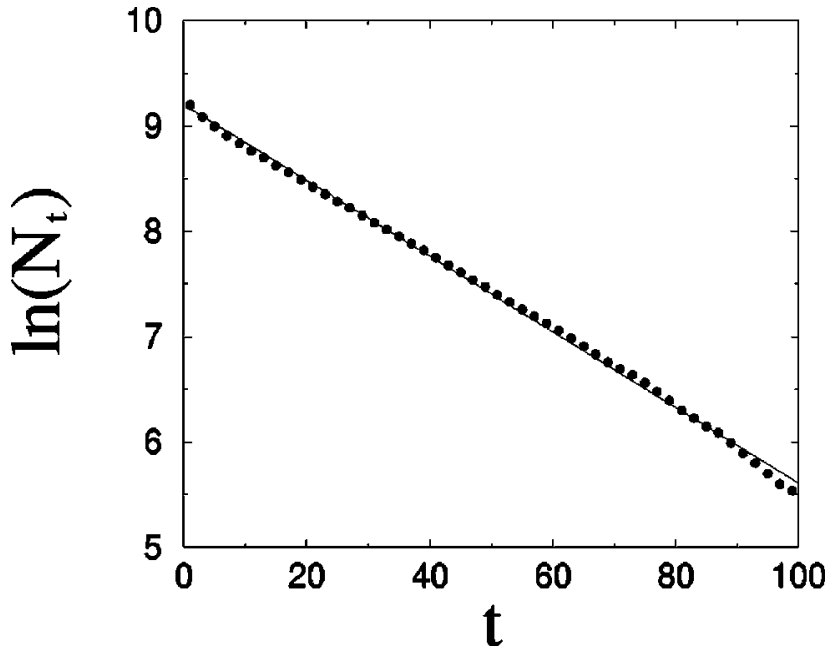

FIG. 10. Exponential decay of $N_{t}$, the number of trajectories inside the restraining region at time $t$, as a function of $t$. The inverse of the slope of the fitted line gives the average exit time $\tau \approx 27.9$.
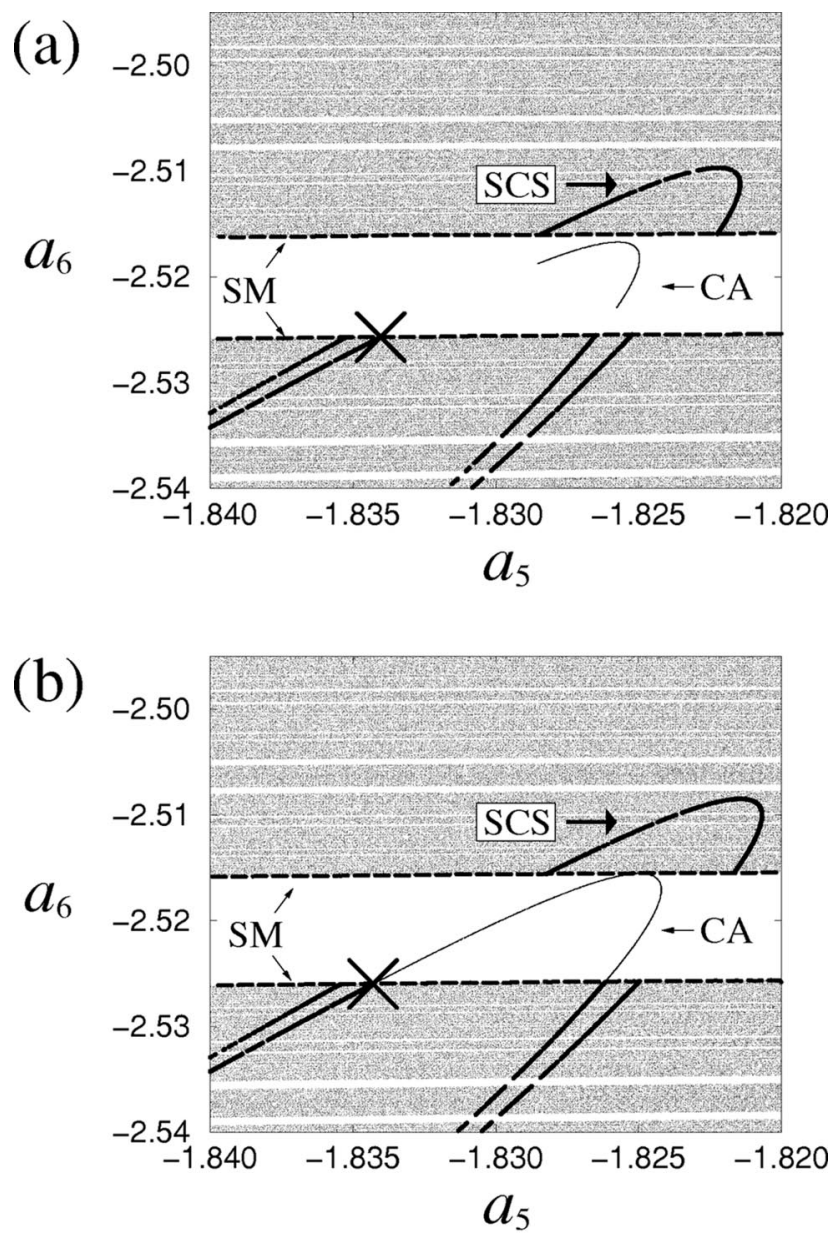

FIG. 11. Plots of the upper branch of the CA (light line), the SCS (dark lines) and its stable manifolds (dots in the background): (a) before the interior crisis, at $\nu=0.0299211$; and (b) at the interior crisis, $\nu$ $=0.02992021$. The cross denotes one of the Poincare points of the p-3 mediating unstable periodic orbit. The dashed lines represent segments of the boundary between the band region and the surrounding region, given by the SMs of the mediating unstable periodic orbit. 


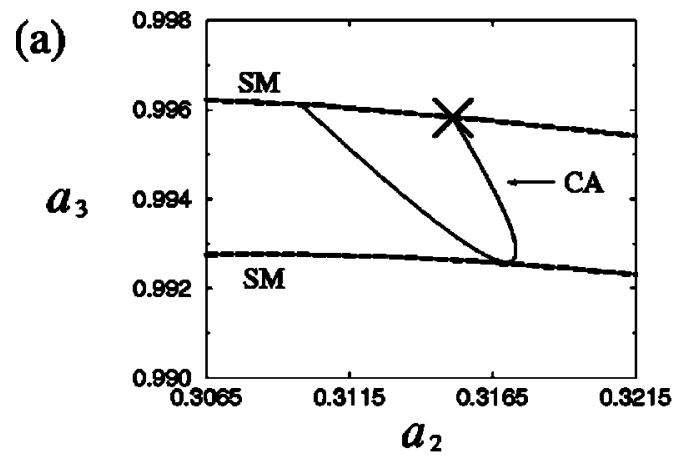

(c)

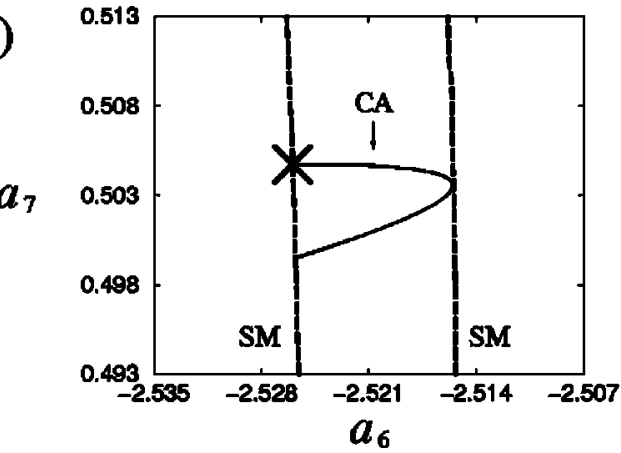

(b)

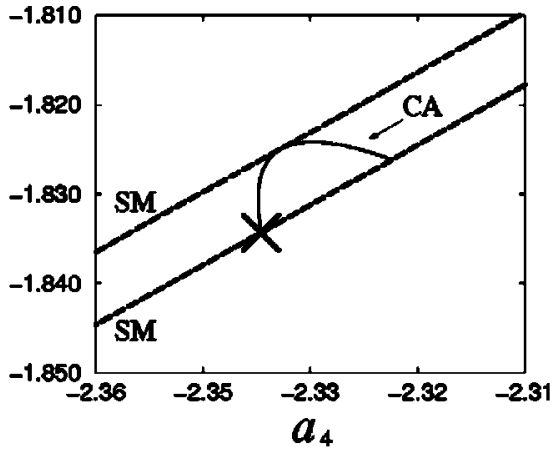

(d)

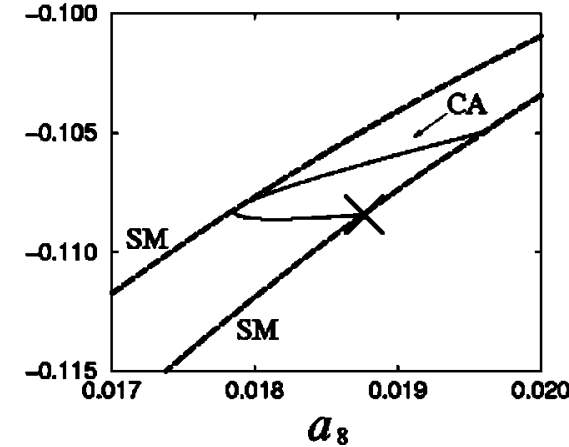

FIG. 12. Four different two-dimensional projections showing the homoclinic tangency between one of the three branches of the CA and the SM (dashed lines) of the mediating unstable periodic orbit (cross) at the interior crisis, $\nu_{\mathrm{IC}}=0.02992021$.

SCS has a large gap between the two dashed lines, and many other smaller gaps, or discontinuities. Its gaps are due to the horizontal white spaces in the background, which reflect the fractal structure of the stable manifold of SCS. The fact that SCS always intersects its stable manifold, and its gaps always coincide with the white spaces in the background indicate that the grid was well chosen. Figure 11(b) reveals that at crisis the $S$ chaotic saddle and the chaotic attractor collide. The collision takes place at the mediating orbit, which belongs to SCS. Likewise, the chaotic attractor collides with the stable manifold of SCS.

Recall that the stable manifold of MPO determines the boundary between the band region and the surrounding region. In our system, the mediating orbit has 14 stable eigen- (a)

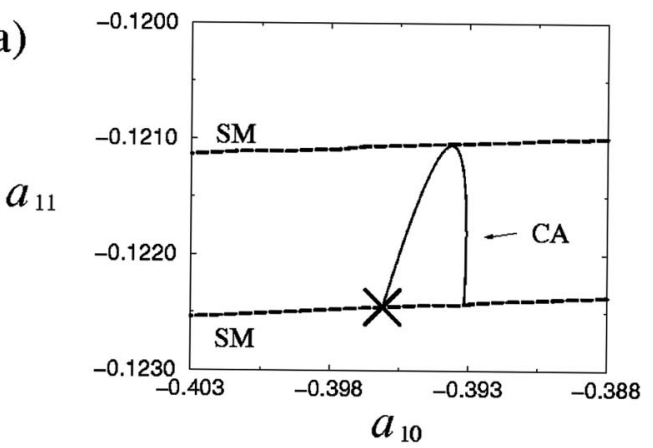

(c)

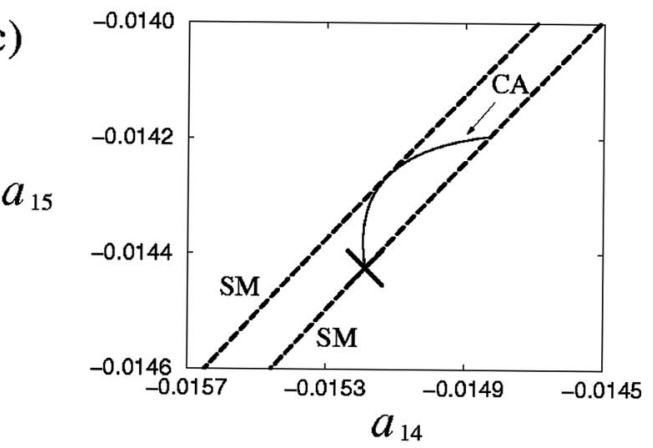

(b)

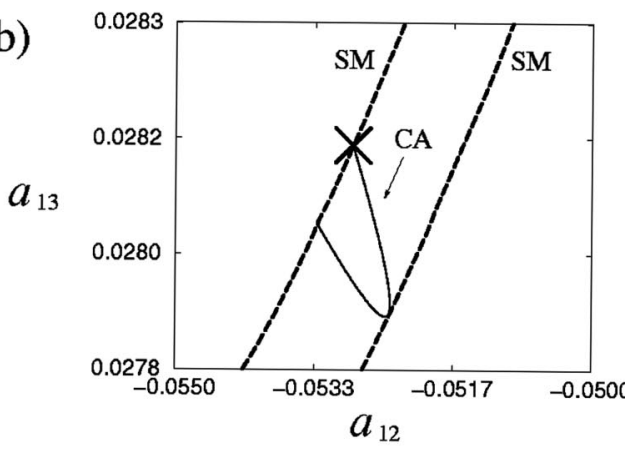

(d)

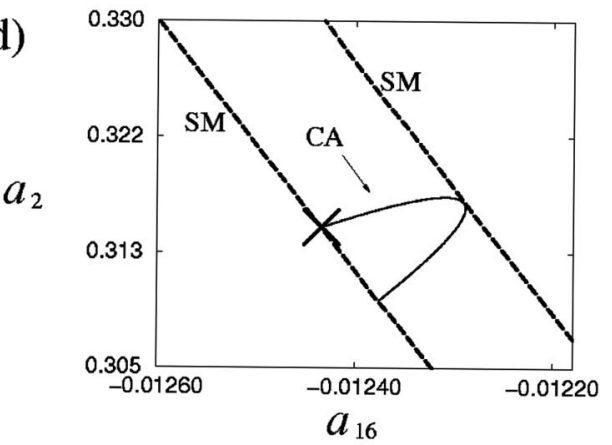

FIG. 13. Four different two-dimensional projections showing the homoclinic tangency between one of the three branches of the CA and the SM (dashed lines) of the mediating unstable periodic orbit (cross) at the interior crisis, $\nu_{\mathrm{IC}}=0.02992021$. 
values (with absolute values less than one), implying that the invariant stable manifold of the mediating orbit has dimension 14. The numerical computation of high-dimensional invariant stable manifolds of an unstable periodic orbit is a highly complicated task. Recent works have presented the computation of two-dimensional invariant manifolds. ${ }^{39-42}$ The computation of stable manifolds of much higher dimensions, as in our case, is still a challenging task. However, we can approximate one-dimensional projections of segments of the stable manifold (SM) of the mediating orbit close to the tangency points using the stable manifold of SCS, to determine the local boundary between regions $B$ and $S$, as shown by the dashed lines in Fig. 11. This can be done since the stable manifold of SCS and the stable manifold of any UPO on SCS are practically indistinguishable. ${ }^{20,33}$ Using this method we reveal in Fig. 11(b) the tangency between the chaotic attractor and the stable manifold of MPO. At crisis, the chaotic attractor is the closure of one branch of the unstable manifold of MPO. ${ }^{23}$ Thus, we have demonstrated in Fig. 11(b) the homoclinic tangency between the stable and unstable manifolds of MPO. Although Fig. 11(b) shows only a two-dimensional projection $\left(a_{5}, a_{6}\right)$ of the phase space, the collision can be observed in projections onto any choice of Fourier modes, as seen in Figs. 12 and 13.

\section{DEFINING THE RESTRAINING REGION}

Both the sprinkler and the PIM triple algorithms, used in the previous sections, require the definition of a restraining region, which is a region of the phase space which contains a chaotic saddle and no attractor. The definition of such regions can be a complex task in a high-dimensional phase space. We consider different strategies for different situations.

For the values of $\nu$ for which the attractor is a periodic orbit the restraining region can be defined as the phase space minus a set of small spheres around the periodic points of the attractor. In order to increase computation speed it is enough to cover just one of the periodic points. The PIM triple algorithm looks for a trajectory that stays forever outside the sphere, that is, at an Euclidean distance larger than a specified radius from the periodic point.

Inside the periodic window, after the sequence of perioddoubling bifurcations, the attractor becomes chaotic and is localized in three separate branches on the Poincare plane. To define the restraining region it is necessary to cover one of the branches without covering parts of the surrounding chaotic saddle. Figure 14 shows the situation at the interior crisis point $\nu_{\mathrm{IC}}$, when the CA (black lines) touches the surrounding chaotic saddle SCS (gray lines). For this case, it is possible to isolate one of the branches of the attractor by covering it with a $15 \mathrm{D}$ box whose projection onto the plane $\left(a_{5}, a_{6}\right)$ is shown in Fig. 14(b), an enlargement of the rectangular region in Fig. 14(a). The upper-right corner of the box is defined by the coordinates of the mediating unstable periodic orbit. The lower-left corner can be obtained from the chaotic attractor. A long chaotic trajectory is generated on the attractor and the point with the lowest $a_{5}$ or $a_{6}$ value is taken as the lower-left corner of the rectangle. Projections (a)

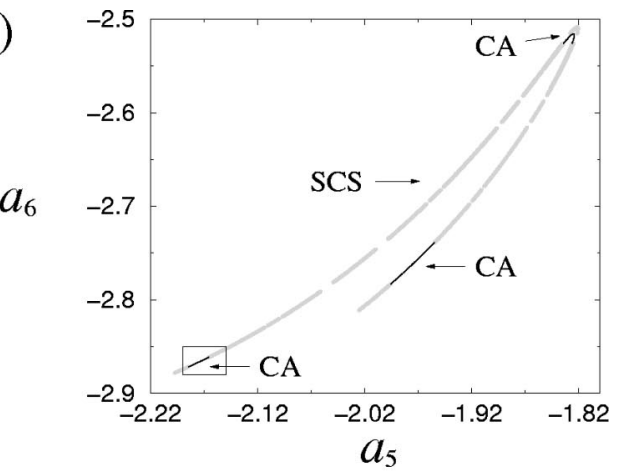

(b)

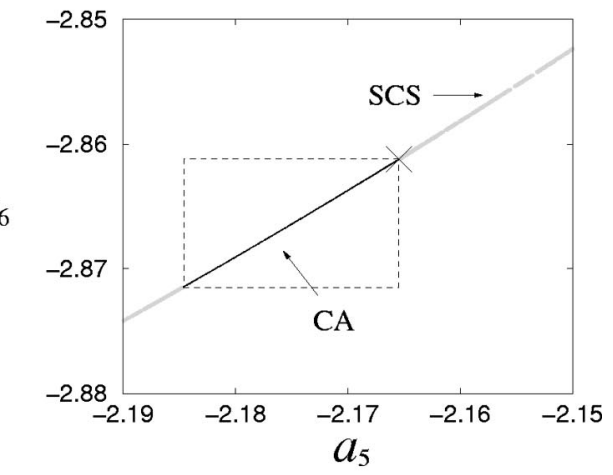

(c)

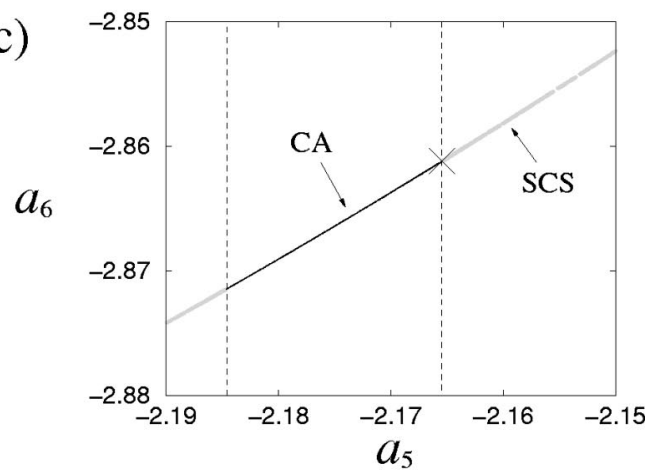

FIG. 14. (a) SCS (gray) and CA (black) at crisis $\nu_{\mathrm{IC}}$; (b) enlargement of the rectangular region in (a) showing the definition of the restraining region with a box covering one of the branches of CA; (c) simpler restraining region, using only $a_{5}$ to determine if a point is in the band region or not.

onto different planes show similar pictures. The surrounding chaotic saddle is found as a straddle trajectory that never enters the box. The box created with this simple method contains this branch of CA due to its particular shape. Different branches could require boxes constructed in different ways. Alternatively, other types of covers can be used, such as sets of spheres, with the care that these spheres do not cover parts of the surrounding region. We have tested several forms of restraining regions, all of which produced similar chaotic saddles. In our particular case, we find that the simpler restraining region can be defined by considering only the $a_{5}$ axis, as in Fig. 14(c). Since the two vertical dashed lines in Fig. 14(c) do not intersect other parts of the chaotic sets, they can be used to identify numerically the points falling into this branch of the chaotic attractor.

After the interior crisis it is not easy to identify the band and surrounding regions. However, for $\nu<\nu_{\mathrm{IC}}$, but still close to the crisis point IC, BCS is localized in three separate 

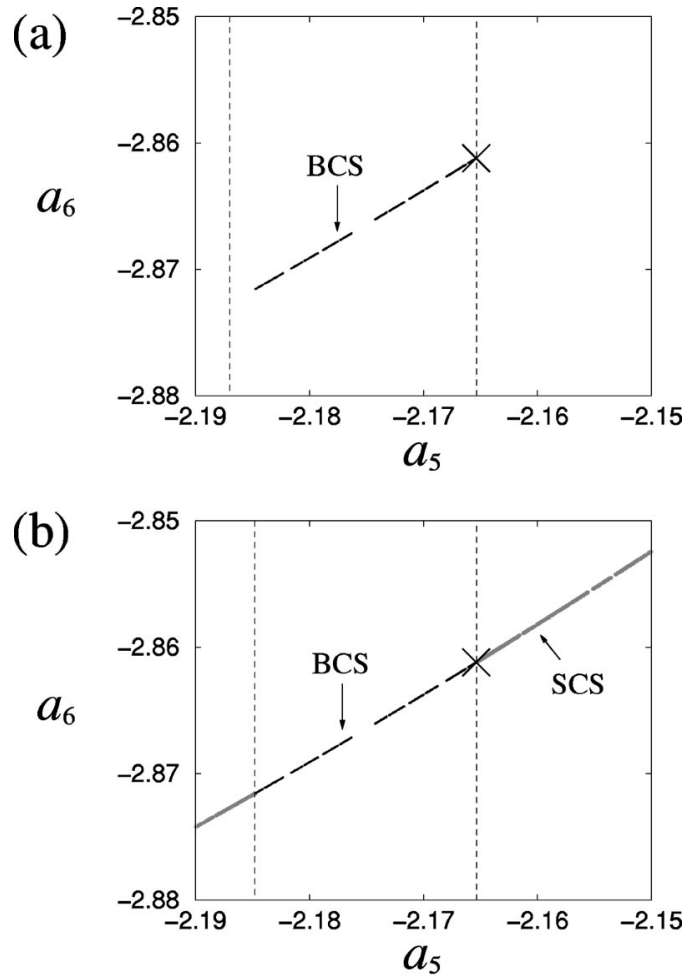

FIG. 15. (a) One of the three branches of the BCS after crisis, at $\nu$ $=0.02992006$. The dashed vertical lines determine the approximate position of the band region. BCS is found as a PIM triple orbit which always returns to this band at every three iterations of the Poicare map; (b) once BCS is found, the left vertical line is adjusted and SCS (gray) is found as a PIM triple orbit which never enters the band region.

regions of the phase space previously occupied by the precrisis band chaotic attractor. BCS can be found by using the PIM triple algorithm to determine a chaotic straddle trajectory that always, at every third iteration (according to the number of bands of the $B$ region), returns to a given restraining region $R$. This region $R$ is defined slightly larger than one of the precrisis bands. Such a region is depicted in Fig. 15(a), where the position of MPO (cross) can still be used to accurately determine the right side of $R$. The left side is somewhat to the left of the band region at crisis [left vertical line in Fig. 14(c)]. The left side of $R$ does not have to be precisely determined to find BCS. Once BCS is found, it can be used to find the best position for the left side of $R$, in order to find SCS as a PIM triple orbit that never enters the region $R$, which is occupied by BCS [Fig. 15(b)].

\section{CONCLUSIONS}

We have studied the nonlinear dynamics of chaotic saddles in a high-dimensional dynamical system exemplified by the Kuramoto-Sivashinsky equation. We presented a method that uses simple linear interpolations between points on the chaotic sets to choose an appropriate grid to visualize a homoclinic tangency in a high-dimensional phase space. Since the grid must be tangent to every point on the chaotic attractor in a certain region of interest, the direct applicability of this method is still limited to cases in which the invariant chaotic sets do not have many expanding directions. More research is needed in order to extend this technique for other situations. Nevertheless, many systems exhibit lowdimensional chaotic attractors in high-dimensional phase spaces, with sequences of bifurcations and crises similar to the cases discussed in this paper, and it should be possible to apply the techniques explained here in such systems. Examples of numerical analysis of partial differential equations which exhibit low-dimensional chaos include a model for the nonlinear evolution of low-frequency magnetohydrodynamic oscillations in plasmas, ${ }^{43}$ a generic model for the propagation of nonlinear waves in forced, spatially extended medium, ${ }^{44}$ and a model for semiconductor devices. ${ }^{45}$ Experimental examples include a magnetoelastic ribbon, ${ }^{46}$ electronic circuits, ${ }^{47}$ a dripping faucet, ${ }^{48}$ experiments with air bubbles, ${ }^{49}$ and lasers. ${ }^{50}$

\section{ACKNOWLEDGMENT}

This work is supported by FAPESP (Brazil).

${ }^{1}$ J. M. Hyman and B. Nicolaenko, Physica D 18, 113 (1986).

${ }^{2}$ R. Temam, Infinite-Dimensional Dynamical Systems in Mechanics and Physics (Springer, New York, 1988).

${ }^{3}$ M. C. Cross and P. C. Hohenberg, Rev. Mod. Phys. 65, 851 (1993).

${ }^{4}$ P. Holmes, J. L. Lumley, and G. Berkooz, Turbulence, Coherent Structures, Dynamical Systems and Symmetry (Cambridge University Press, Cambridge, 1996).

${ }^{5}$ F. Christiansen, P. Cvitanović, and V. Putkaradze, Nonlinearity 10, 55 (1997).

${ }^{6}$ T. Bohr, M. H. Jensen, G. Paladin, and A. Vulpiani, Dynamical Systems Approach to Turbulence (Cambridge University Press, Cambridge, 1998).

${ }^{7}$ K. He, Phys. Rev. Lett. 80, 696 (1998).

${ }^{8}$ C. Grebogi, E. Ott, and J. A. Yorke, Phys. Rev. Lett. 48, 1507 (1982)

${ }^{9}$ C. Grebogi, E. Ott, and J. A. Yorke, Physica D 7, 181 (1983).

${ }^{10}$ Y.-C. Lai and R. L. Winslow, Phys. Rev. Lett. 74, 5208 (1995).

${ }^{11}$ R. Braun and F. Feudel, Phys. Rev. E 53, 6562 (1996).

${ }^{12}$ S. Bleher, E. Ott, and C. Grebogi, Phys. Rev. Lett. 63, 919 (1989).

${ }^{13}$ E. E. N. Macau and I. L. Caldas, Phys. Rev. E 65, 026215 (2002).

${ }^{14}$ S. W. McDonald, C. Grebogi, E. Ott, and J. A. Yorke, Physica D 17, 125 (1985).

${ }^{15}$ P. M. Battelino, C. Grebogi, E. Ott, and J. A. Yorke, Physica D 32, 296 (1988).

${ }^{16}$ A. Péntek, T. Zontán, T. Tél, C. Grebogi, and J. A. Yorke, Phys. Rev. E 51, 4076 (1995).

${ }^{17}$ K. G. Szabó and T. Tél, Phys. Lett. A 196, 173 (1994).

${ }^{18}$ K. G. Szabó, Y.-C. Lai, T. Tél, and C. Grebogi, Phys. Rev. Lett. 77, 3102 (1996).

${ }^{19}$ K. G. Szabó, Y.-C. Lai, T. Tél, and C. Grebogi, Phys. Rev. E 61, 5019 (2000).

${ }^{20}$ E. L. Rempel and A. C.-L. Chian, Int. J. Bifurcation Chaos Appl. Sci. Eng. (in press).

${ }^{21}$ E. L. Rempel and A. C.-L. Chian, Phys. Lett. A 319, 104 (2003).

${ }^{22}$ F. A. Borotto, A. C.-L. Chian, and E. L. Rempel, Int. J. Bifurcation Chaos Appl. Sci. Eng. (in press).

${ }^{23}$ A. C.-L. Chian, E. L. Rempel, E. E. Macau, R. R. Rosa, and F. Christiansen, Phys. Rev. E 65, 035203(R) (2002).

${ }^{24}$ Y. Kuramoto and T. Tsuzuki, Prog. Theor. Phys. 55, 356 (1976).

${ }^{25}$ G. I. Sivashinsky, Acta Astron. 4, 1177 (1977).

${ }^{26}$ R. E. LaQuey, S. M. Mahajan, P. H. Rutherford, and W. M. Tang, Phys. Rev. Lett. 34, 391 (1975).

${ }^{27}$ G. I. Sivashisnky and D. M. Michelson, Prog. Theor. Phys. 63, 2112 (1980).

${ }^{28}$ A. J. Babchin, A. L. Frenkel, B. G. Levich, and G. I. Sivashinsky, Phys. Fluids 26, 3159 (1983).

${ }^{29}$ M. Münkel and F. Kaiser, Physica D 98, 156 (1996).

${ }^{30}$ D. Gottlieb and S. A. Orszag, Numerical Analysis of Spectral Methods: Theory and Applications (Society for Industrial and Applied Mathematics, Philadelphia, 1977).

${ }^{31}$ K. T. Alligood, T. D. Sauer, and J. A. Yorke, Chaos: An Introduction to Dynamical Systems (Springer, New York, 1996).

${ }^{32}$ H. E. Nusse and J. A. Yorke, Physica D 36, 137 (1989). 
${ }^{33}$ E. M. Ziemniak, C. Jung, and T. Tél, Physica D 76, 123 (1994).

${ }^{34}$ H. Kantz and P. Grassberger, Physica D 17, 75 (1985).

${ }^{35}$ G.-H. Hsu, E. Ott, and C. Grebogi, Phys. Lett. A 127, 199 (1988).

${ }^{36}$ J. H. Curry, in Global Theory of Dynamical Systems, edited by Z. Nitecki and C. Robinson (Springer, New York, 1979), pp. 111-120.

${ }^{37}$ P. Frederickson, J. L. Kaplan, E. D. Yorke, and J. A. Yorke, J. Diff. Eqns. 49, 185 (1983).

${ }^{38}$ M. Dhamala and Y.-C. Lai, Phys. Rev. E 59, 1646 (1999).

${ }^{39}$ B. Krauskopf and H. M. Osinga, J. Comput. Phys. 146, 404 (1998).

${ }^{40}$ W. J. Beyn and W. Kless, Numer. Math. 80, 1 (1998).

${ }^{41}$ B. Krauskopf and H. M. Osinga, Chaos 9, 768 (1999).

${ }^{42}$ M. E. Johnson, M. S. Jolly, and I. G. Kevrekidis, Int. J. Bifurcation Chaos Appl. Sci. Eng. 11, 1 (2001).

${ }^{43}$ S. Ghosh and K. Papadopoulos, Phys. Fluids 30, 1371 (1987).
${ }^{44}$ Y.-C. Lai, E. M. Bollt, and Z. Liu, Chaos, Solitons Fractals 15, 219 (2003).

${ }^{45}$ S. Bose, A. Wacker, and E. Schöll, Phys. Lett. A 195, 144 (1994).

${ }^{46}$ W. L. Ditto, S. Rauseo, R. Cawley, C. Grebogi, G.-H. Hsu, E. Kostelich, E. Ott, H. T. Savage, R. Segnan, M. L. Spano, and J. A. Yorke, Phys. Rev. Lett. 63, 923 (1989).

${ }^{47}$ D. C. Hamill, J. H. B. Deane, and D. J. Jefferies, IEEE Trans. Power Electron. 7, 25 (1992).

${ }^{48}$ A. Tufaile, W. M. Goncalves, and J. C. Sartorelli, Phys. Lett. A 255, 58 (1999).

${ }^{49}$ A. Tufaile and J. C. Sartorelli, Phys. Lett. A 287, 74 (2001).

${ }^{50}$ S. Wieczorek, T. B. Simpson, B. Krauskopf, and D. Lenstra, Phys. Rev. E 65, 045207(R) (2002). 